\title{
Valoración de las funciones mentales y la comunicación en adultos mayores mapuches y no mapuches en áreas rurales del sur de Chile
}

\author{
Rebeca Mella Ma , María Alvear Aa , Berta Carrillo Va, \\ Victor Caire V.
}
Mental and communication disorders in Mapuche and non Mapuche elderly subjects in rural communities in southern Chile

Background: The main predictors of functional impairment in the elderly are alterations in mental or communication functions. Aim: To study mental and communication functions in rural elderly subjects of Mapuche and non Mapuche origin. Patients and methods: Elderly subjects coming from a rural Mapuche community and a non Mapuche community were studied. Subjects were interviewed at their homes. The communication and mental function assessments of the Functional Autonomy Measurement System were applied. Results: Fifty one Mapuche and 49 non Mapuche subjects with a mean age of $71 \pm 7$ and $74 \pm 8$ years respectively, were studied. Fifty four percent were female and $31 \%$ were illiterate. Twenty six percent had impairment in mental functions. The item with the highest difficulty was memory. The visual function was the most severely impaired among communication items. Mapuche elderly subjects had significantly higher degrees of impairment in mental and communication functions. Conclusions: There is a higher degree of mental and communication impairment among rural Mapuche elderly subjects than in their non Mapuche counterparts (Rev Méd Chile 2003; 31: 1257-65).

(Key Words: Communication disorders; Frail elderly; Indians, South American; Memory disorders)

Recibido el 11 de diciembre, 2002. Aceptado en versión corregida el 18 de agosto, 2003. Departamento de Salud Pública, Facultad de Medicina, Universidad de La Frontera, Temuco.

aEnfermera Universitaria.

Correspondencia a: Rebeca Mella M. Dirección postal Manuel Montt 112, Temuco. Facultad de Medicina UFRO. E mail: rmella@ufro.cl 
L a proporción de personas mayores de 60 años está aumentado en el mundo. Se prevé que en el año 2025 el número de personas de esa edad habrá aumentado con respecto al año 1970 en $28,1 \% 1,8$. Los adultos mayores (AM) aumentaron catorce veces en el siglo XX y se duplicarán en el siglo XXI (2000: 10\% - 2035: 20\%). A partir del 2035 los AM superarán a los menores de 15 años. La esperanza de vida en el mundo alcanza en la actualidad a 66 años y en Chile a 76 años ${ }^{1,2}$.

Los antecedentes demográficos muestran que Chile está en etapa de transición avanzada de envejecimiento, con fecundidad y mortalidad baja.

El avance de la transición demográfica de la población se reafirma con los resultados del censo del año 2002. Así, mientras en 1992, la población menor de 15 años representaba 29,4\%, en enero de 2002 fue 25,7\%. En tanto, las personas de 60 años o más, aumentaron de 9,8 a $11,4 \%$ en el año 2002.

Desde el punto de vista de estos indicadores demográficos es importante señalar algunos aspectos tales como el promedio de vida alcanzado por el hombre y la mujer, el que ha aumentado en los últimos años como consecuencia del desarrollo industrial, progresos en medicina, calidad en la atención de salud y control de la natalidad entre otros. Por tal motivo, el desarrollo demográfico presenta una prevalencia ascendente de adultos mayores $^{3}$.

En este contexto, evaluando las proyecciones de mayor envejecimiento poblacional se hace indispensable considerar aspectos como los sociales y, entre estos, los étnicos, que debieran ser estudiados para intervenir con criterio de riesgo.

En Chile, 19,7\% de la población se reconoce perteneciente a la etnia mapuche. La Región de la Araucanía es la cuna de ésta con 38\% y en la comuna de Nueva Imperial, asciende a $53 \% 3$. Considerando básicamente la relación que las influencias ambientales ejercen sobre el proceso de envejecimiento, es que este estudio se diseñó en un área rural, ámbito donde es posible encontrar en mayor grado de pureza, la práctica de costumbres y creencias identificadas como propias por la cultura mapuche. A pesar que la sociedad mapuche ha sido estudiada desde diversas perspectivas, la ancianidad es un tema ausente. Lo poco que hay, forma parte de tratados más amplios sobre temas como la religiosidad, el grupo doméstico, la muerte, etc., por lo que las características individuales y sociales que adquiere esta etapa de la vida entre los mapuches carece de un tratamiento profundo. Pareciera, sin embargo, que los mapuches comparten gran parte de las características de las sociedades tradicionales rurales en relación a la valoración de la ancianidad ${ }^{6}$.

El trabajo, la familia, el rol social, están determinados por una cultura donde se valora la ancianidad como una etapa importante de la vida de un hombre o de una mujer. No obstante, los cambios que se están produciendo al interior de este grupo étnico, están marcando nuevas formas de relaciones sociales y de construcción de diferencias generacionales ${ }^{6}$.

En la cultura mapuche se relaciona la vejez con cambios funcionales. Es una etapa cuando los mapuches sufren cambios fisiológicos que no se diferencian de los conocidos como propios de la tercera edad. Es un momento de la vida cuando la persona es valorada por el conocimiento que ha alcanzado durante toda la vida, los jóvenes acuden donde ellos (as) para escucharlos (as) y la comunidad cuida y aprecia la labor de orientación que los ancianos cumplen en la sociedad.

Dentro de la familia, el padre es la figura central y que después de su muerte, su rol autoritario como organizador de las actividades familiares, sólo puede ser alcanzado por la madre o su hijo mayor. Los hijos no pueden contradecir o desobedecer a sus padres, su autoridad permanece inclusive con el paso de la edad. A diferencia de los hombres, las viudas tienden a ganar mayor poder persuasivo a medida que van envejeciendo. La vejez es considerada un estado de vida en que se esperan retribuciones de los hijos y nietos. El respeto hacia el anciano también se centra en una cuestión económica, porque mientras el paterfamilias viva y controle sus tierras, sus hijos trabajan una parte solo con su consentimiento y en tal caso, bajo su control económico. Los hijos respetan al padre en las actividades rituales, ya que como miembro más viejo del linaje posee una importante posición religiosa y moral. Esta relación contrarresta el puro interés económico entre los miembros de la familia, ya sea que vivan bajo el mismo techo en una familia extensa compuesta o dispersos en una misma reducción. La superiori- 
dad moral se aprecia en que son los ancianos a quienes se les consulta sobre las decisiones importantes para la comunidad, lo que en muchos casos se refleja en la figura del Lonko (Cabeza) ${ }^{6}$.

En la cultura mapuche se es viejo cuando ya no se hace lo que antes se hacía. Cuando el adulto mayor ya no puede realizar, entre otras cosas, tareas o actividades para la mantención de la familia ${ }^{4}$; descripción que se puede comparar con las líneas generales de la teorías psicosociales, las que indican que el envejecimiento puede describirse como el resultado de la falta de uso de las habilidades adquiridas anteriormente; la consecuencia del desgaste aleatorio; un cambio en la capacidad de adaptación por variables ambientales; la pérdida de recursos internos y externos; el resultado de la influencia genética sobre la duración de la vida, que puede provocar cambios en las características psicosociales individuales, o la consecuencia de la elección realizada por el individuo y la creación de nuevas situaciones nunca utilizadas ${ }^{5}$.

La aproximación general de las teorías psicosociológicas del envejecimiento, indican que el envejecimiento psicológico se caracteriza sobre todo por los cambios conductuales $\mathrm{y}$, estrechamente relacionados con los cambios psicológicos y biológicos, se encuentran los cambios sociológicos, que se relacionan con las influencias ambientales.

Kane define función como la resultante entre las capacidades físicas por tratamiento por motivación, partido por ambiente físico y social ${ }^{7}$.

La capacidad funcional es el resultado de las capacidades innatas del individuo y el ambiente que sustenta dichas capacidades. Se incorpora aquí el término suncionalidad», el que necesariamente debe incluirse en el diagnóstico integral del AM, además de los clásicos indicadores de morbilidad, mortalidad, diagnóstico funcional, que corresponde a uno de los elementos de diagnóstico más importantes que se han introducido en la geriatría 7 .

En los adultos mayores, la pérdida de función corresponde a la principal manifestación de la enfermedad. Esta pérdida de función se puede detectar clínicamente por la pérdida de la autonomía y la aparición de dependencia que, poco a poco, va a limitar la calidad de vida. Esta pérdida de autonomía, afecta inicialmente a las actividades complejas avanzadas, pero si se mantiene en el tiempo y no se actúa sobre ella, progresa y llega a afectar la autonomía para actividades más elementales o clásicas ${ }^{7}$.

Parrochia propone algunos conceptos en relación a lo que se podría considerar una vejez satisfactoria, entre otros: estado mental alerta, compatible con una correcta orientación témporo espacial y una fluida comunicación interpersonal y colectiva ${ }^{9}$.

La OMS señala como factores de riesgo predictores de declinación funcional del adulto mayor a elementos de la comunicación tales como: no ver bien (a pesar de usar lentes); no escuchar bien y en las funciones mentales: la memoria, no poder quedarse solo en la casa; orientación, etc ${ }^{10}$.

El objetivo de nuestro trabajo fue determinar la prevalencia de discapacidad o dependencia en las funciones mentales y comunicación en un grupo de adultos de etnia mapuche y no mapuche, residentes en un área rural.

\section{MATERIAL Y MÉTODO}

Tipo de estudio. Comparativo, transversal cuya unidad de análisis estuvo constituida por adultos mayores de dos comunidades rurales, una mapuche (con dos apellidos mapuches, residente en una comunidad mapuche por 10 años o más, de habla mapudungun) y otra no mapuche (sin apellidos mapuches, residentes en una comunidad rural por más de 10 años, de habla castellana) de la comuna de Nueva Imperial, IX región, Chile.

Población. Universo, 170 AM. El número de AM a los cuales se les aplicó el instrumento de evaluación fue 100. Para la recolección de datos de esta investigación se utilizó el instrumento Sistema de Medición de la Autonomía Funcional (SMAF). modificado ${ }^{11}$.

El SMAF contempla cinco áreas fundamentales de la capacidad funcional, las cuales son cercanamente similares a la clasificación de la OMS para incapacidad. Mide la capacidad funcional en cinco áreas:

1. Actividades de la vida diaria (AVD): en cinco items. 2. Movilidad: seis items. 3. Comunicación: tres items. 4. Función mental: cinco items. 5. 
Actividades instrumentales de la vida diaria: ocho items.

El instrumento Sistema de Medición de la Autonomía Funcional (SMAF) aplicado, fue validado en Chile, en población urbana y rural en Puerto Montt en 199712 .

Fundado en el interés de evaluar la autonomía en adultos mayores rurales se decidió aplicar las áreas 3 y 4 correspondientes a comunicación y funciones mentales (Anexo 1).

El instrumento fue aplicado en el domicilio de los adultos mayores, a través de una visita domiciliaria por personal capacitado previamente en la aplicación de éste y para la población mapuche se utilizó apoyo bilingüe (mapudungun).

Estadística: Análisis de los datos. Para el análisis de los datos, la comparación entre las mediciones de ambos grupos, se estimó adecuada la prueba exacta de Fisher. El nivel de significación utilizado fue de $5 \%$.

\section{RESULTADOS}

Descriptivos. En la Tabla 1 observamos el comportamiento de las variables sociodemográficas: $51 \%$ de los AM se ubicaron entre 65 y 70 años, $54 \%$ de sexo femenino, $36 \%$ se ubica en el nivel de instrucción entre $4^{\circ}$ y $8^{\circ}$ básico y $31 \%$ en el nivel de analfabetos. El 56\% recibía apoyo de su pareja, $37 \%$ de otros familiares como hijos o nietos, y $11 \%$ vivía solo, sin apoyo social. El 50\% se declaró perteneciente a la etnia mapuche.

Comparativos: La Tabla 3, refleja una media de 71,2 años en los AM no mapuches, y una media levemente superior (74 años) en los AM mapuches. Para efecto de comparación de los grupos en estudio, la diferencia en el promedio de edad no fue significativa.

En la Tabla 4 podemos observar la distribución según sexo, tanto en los AM mapuches como en los no mapuches hay una mayor proporción de mujeres en el grupo en estudio. Fisher 0,506, p $>0,05$. La diferencia entre ambos grupos étnicos en relación al comportamiento de esta variable no fue significativa.
Tabla 1. D istribución porcentual de los AM según variables sociodemográficas

\begin{tabular}{|c|c|c|}
\hline Variables & $\mathrm{n}$ & $\%$ \\
\hline \multicolumn{3}{|l|}{ Edad (años) } \\
\hline $65-70$ & 51 & 51 \\
\hline 71-75 & 16 & 16 \\
\hline $76-80$ & 14 & 14 \\
\hline 81 y más & 19 & 19 \\
\hline \multicolumn{3}{|l|}{ Sexo } \\
\hline Hombres & 46 & 46 \\
\hline Mujeres & 54 & 54 \\
\hline \multicolumn{3}{|l|}{ Nivel de Instrucción } \\
\hline Analfabeto & 31 & 31 \\
\hline 1ํ-3ํㅡ básico & 26 & 26 \\
\hline $4^{0}-8^{0}$ básico & 36 & 36 \\
\hline Media incompleta & 07 & 07 \\
\hline \multicolumn{3}{|l|}{ Apoyo social } \\
\hline Vive solo & 11 & 11 \\
\hline Vive con pareja & 56 & 56 \\
\hline Con familia & 37 & 37 \\
\hline Otros & 06 & 06 \\
\hline \multicolumn{3}{|l|}{ Etnia } \\
\hline Mapuche & 50 & 50 \\
\hline No mapuche & 50 & 50 \\
\hline
\end{tabular}

Tabla 2. D istribución de la población según edad, comunidades mapuches y no mapuches, comuna de $\mathrm{N}$ ueva Imperial, IX región

\begin{tabular}{|c|c|c|c|c|}
\hline \multirow{2}{*}{ Edad (años) } & \multicolumn{2}{|c|}{ Mapuche } & \multicolumn{2}{|c|}{ No Mapuche } \\
\hline & $\mathrm{n}^{0}$ & $\%$ & $\mathrm{n}^{0}$ & $\%$ \\
\hline $65-74$ & 30 & 58,8 & 30 & 61,24 \\
\hline $75-80$ & 10 & 19,7 & 12 & 24,48 \\
\hline 81-98 & 11 & 21,5 & 7 & 14,28 \\
\hline Total & 51 & 100 & 49 & 100 \\
\hline
\end{tabular}

Tabla 3. D istribución de la población por promedio de edad, comunidades mapuches y no mapuches

\begin{tabular}{|lcc|}
\hline Comunidad & (Media) años & (DE) años \\
\hline No Mapuche & 71,18 & 7,22 \\
Mapuche & 73,96 & 8,55 \\
\hline
\end{tabular}

$\mathrm{p}>0,05$. 
El 6,1\% de la población no mapuche tenía enseñanza media, y 2,0\% en los mapuches, encontrandose un mayor porcentaje de analfabetismo en la población mapuche $(39,2 \%)$ que en la no mapuche (22,5\%). Fisher $0,344, p>0,05$. No se observaron diferencias significativas en el nivel de instrucción en la población mapuche y no mapuche (Tabla 5).

La Tabla 6 muestra que $70 \%$ de los AM son independientes en el área de las funciones mentales. La Figura 1 muestra las diferencias en las proporciones según grupos en estudio: los AM

Tabla 4. D istribución de la población según sexo, mapuche y no mapuche

\begin{tabular}{|lcccccc|}
\hline Comunidad & \multicolumn{2}{c}{ Sexo Fem } \\
& $\mathrm{n}^{\mathbf{0}}$ & $\%$ & \multicolumn{2}{c|}{ Sexo Masc } \\
no & $\%$ & \multicolumn{2}{c|}{ Total } \\
$\mathrm{n}^{\mathbf{0}}$ & $\%$ \\
\hline No mapuche & 26 & 54,6 & 23 & 46,40 & 49 & 100 \\
Mapuche & 28 & 55,4 & 23 & 45,10 & 51 & 100 \\
Total & 54 & 100,0 & 46 & 100,0 & 100 & 100 \\
\hline
\end{tabular}

Fisher's exact $=0,506$. mapuches tienen mayores porcentajes de dependencia, Fisher 0,0080, $p<0,05$. Las diferencias en el nivel de independencia en las funciones mentales entre mapuches y no mapuches fueron significativas.

El item que presentó mayor grado de dificultad fue el de memoria (Figura 4), tanto en mapuches como en no mapuches. Se observó además porcentajes mayores de dependencia en los mapuches, diferencias significativas.

El 57\% de los AM fueron independientes en el área de la comunicación (Tabla 7). La Figura 3 muestra las diferencias en las proporciones según grupos en estudio. Los AM mapuches tienen mayores porcentajes de dependencia, Fisher $0,010, p<0,05$. Las diferencias en el nivel de independencia en comunicación entre mapuches y no mapuches fueron significativas.

El item que presentó mayor grado de dificultad fue el de visión, tanto en mapuches como en los no mapuches (Figura 4). Se observó, además, porcentajes significativos en el item de conversación, donde los AM mapuches tienen porcentajes superiores de dependencia.

Tabla 5. D istribución de la población mapuche y no mapuche según nivel de instrucción

\begin{tabular}{|lrcrrrr|}
\hline Nivel de & \multicolumn{2}{c}{ No Mapuche } & \multicolumn{2}{c}{ Mapuche } & \multicolumn{2}{c|}{ Total } \\
Instrucción & $\mathrm{n}^{\mathbf{0}}$ & $\%$ & $\mathrm{n} \mathbf{n}^{-}$ & $\%$ & $\mathrm{n}^{\mathbf{0}}$ & $\%$ \\
\hline Analfabeto & 11 & 22,45 & 20 & 39,22 & 31 & 31,0 \\
1-30 básico & 15 & 30,9 & 11 & 21,57 & 24 & 26,00 \\
4-8 básico & 20 & 40,82 & 19 & 37,25 & 39 & 39,00 \\
Enseñanza Media & 3 & 6,12 & 1 & 1,96 & 4 & 4,00 \\
Total & 49 & 100,00 & 51 & 100,00 & 100 & 100,00 \\
& & & & & & \\
\hline
\end{tabular}

Fisher's exact $=0,344$.

Tabla 6. D istribución porcentual de los AM según evaluación de las funciones mentales

\begin{tabular}{|lrr|}
\hline AM funciones mentales & $\mathrm{n}$ & $\%$ \\
\hline Independientes & 70 & 70,0 \\
Dependencia leve & 26 & 26,0 \\
Dependencia moderada & 02 & 2,0 \\
Dependencia severa & 02 & 2,0 \\
Total & 100 & 100,0 \\
\hline
\end{tabular}

Tabla 7. D istribución porcentual de los AM según evaluación de la función comunicación

\begin{tabular}{|lrr|}
\hline AM comunicación & $\mathrm{n}$ & $\%$ \\
\hline Independientes & 57 & 57,0 \\
Dependencia leve & 39 & 39,0 \\
Dependencia moderada & 02 & 2,0 \\
Dependencia severa & 02 & 2,0 \\
Total & 100 & 100,0 \\
\hline
\end{tabular}




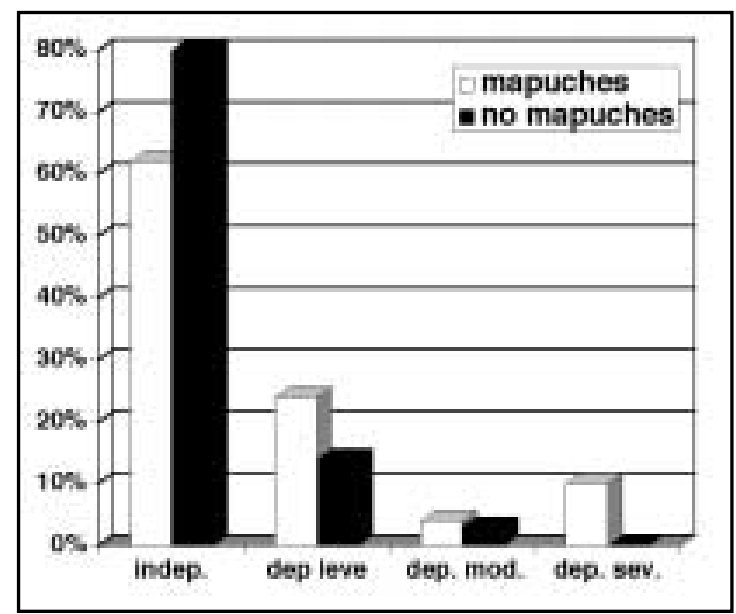

FIgURA 1. Distribución porcentual de los AM según etnia declarada y capacidad en las funciones mentales. $\mathrm{P}<0,05$.

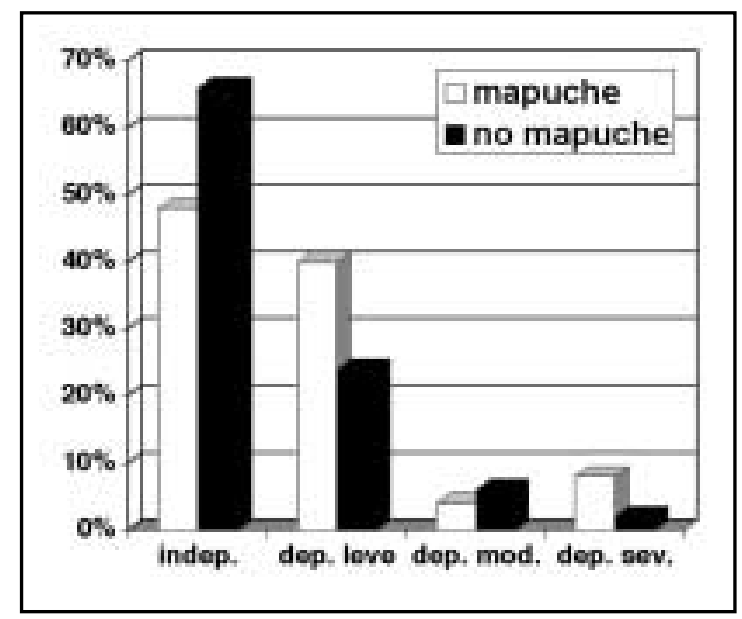

FiguRA 3. Distribución porcentual de los AM según etnia declarada y capacidad en comunicación.

\section{Discusión}

La funciones mentales consideradas predictores de riesgo, son difíciles de definir o evaluar a través de determinadas conductas. En el instrumento aplicado se consideraron 5 conductas, de éstas, la más deficitaria fue la memoria. Sequeira, en su estudio $^{13}$, encontró $29,6 \%$ de dependencia en las funciones mentales. Sin embargo, los items que

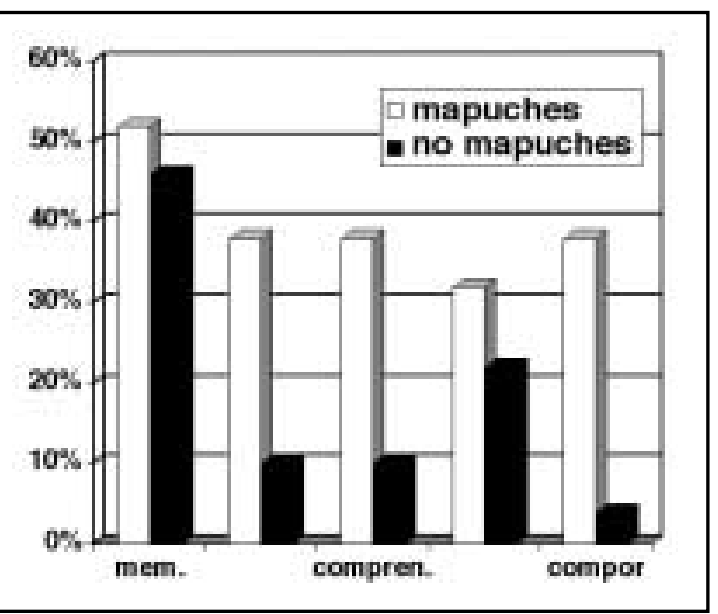

FIgURA 2. Distribución porcentual de dependencia en los items del área de las funciones mentales. Población mapuche y no mapuche.

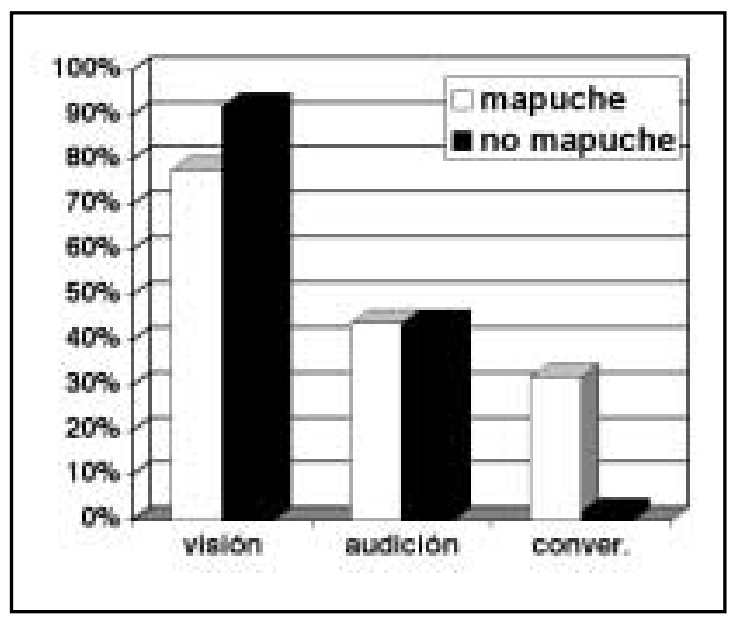

FIgURA 4. Distribución porcentual de dependencia de los itemes del área de comunicación. Población mapuche y no mapuche.

evaluó no correspondían a las conductas evaluadas en nuestro estudio, el que encontró, en general, 26\% de dependencia.

La propuesta que realiza Parrochia, en relación a considerar el estado mental y elementos de la comunicación como indicador de una vejez satisfactoria, parece tomar fuerza ante la conceptualización de las teorías psicológicas. 
En el área comunicación, observamos que un porcentaje considerable de AM son dependientes, siendo el ítem de visión el que presentó mayor dificultad en ambos grupos de estudio. Antecedentes de trabajos realizados en diferentes medios (Europa y Estados Unidos de Norteamérica) muestran que casi la mitad de los ancianos piensan que la pérdida de la visión o audición, cansancio ante pequeños esfuerzos, tristeza o pérdida de la memoria, son manifestaciones normales de la vejez y no síntomas incipientes de: cataratas, tapón de cerumen, insuficiencia cardíaca, depresión o demencia ${ }^{10}$. El 39\% de los AM del presente estudio, manifestó tener discapacidad en esta área. El acceso de los adultos mayores de las áreas rurales a oftalmólogos es muy dificultoso y es habitual que un alto porcentaje de ellos se quede

\section{REFERENCIAS}

1. Instituto Nacional de Estadística. Anuario Demográfico Nacional. Chile, 1997.

2. Ministerio de Salud. Política de Salud para el Adulto Mayor. Chile, 1998.

3. Instituto Nacional de Estadística. Síntesis regional estadística. Chile, IX región, 1999.

4. Oyarce A. Medicinas y Culturas en la Araucanía. Edit. Sudamericana. Santiago, 1995.

5. BuRKe M, WaLSH M. Enfermería Gerontológica. Edit. Harcourt Brace, España. 1998.

6. Huechúan S. El envejecimiento desde una perspectiva cultural. El caso de los mapuches de la Araucanía. Pers-pectiva de Trabajo Social (Universidad Católica Blas Cañas, Santiago, Chile), 1996.

7. Kame R, Ouslander G, Abrass I. Geriatría Clínica. 3a Edic. Edit. Mac Graw-Hill Interamericana. México, 1997.

8. Motus J. La vejez y sus múltiples caras. 1a Edic, Edit. Aurora España, 1985.

9. Parrochia B. Patología del adulto mayor. Bol Hosp San Juan de Dios 1998; 45: 260-7. sin solución de su problema a pesar, incluso, de haber sido diagnosticado. En relación al ítem conversación, que fue el que presentó mayor dependencia en los AM mapuches, Oyarce plantea que en éstos, la actitud de comunicación se observa más contemplativa que expresiva, a medida que el adulto envejece ${ }^{4}$.

Los resultados de este estudio nos permiten reconocer en el ámbito rural, mapuche y no mapuche, diferencias significativas respecto a aspectos de gran relevancia en la vejez. Siendo así, sería recomendable que el nivel primario de atención de salud abordara precozmente el déficit funcional en la población de riesgo, con especial énfasis en lo referente a la salud intercultural, respetando la diversidad socio antropológica.

10. http://www.paho.org/spanish/gou/ce/spp. OPS OMS-36 a sesión del Subcomité de Planificación y Programación Ejecutivo: La Salud y el Envejecimiento. 1998.

11. HÉBERT R. Functional Decline in old age. CMAJ 1997; 157: 1037-45.

12. Casanueva J. Validación del Instrumento Sistema de Medición de la Autonomía funcional. Tesis para optar al grado de Magíster en Ciencias de la Salud con mención en epidemiología clínica. Temuco, Chile, 1998.

13. Sequeira D. Evaluación Funcional. Tesis para optar al Diplomado en Especialidad en Geriatría. Universidad de Concepción, Chile. 1997.

14. Tang Z, Wang H, Meng C. Prevalencia de discapacidad funcional en las AVD y en las AIVD en adultos mayores, Beijin China, AGG, 1999; 22: 115-25.

15. AcuÑa H. Remozar la vejez. OPS. 1982.

16. MEDELín G. Crecimiento y desarrollo del ser humano. 1a edic. OPS, Bogotá, Colombia, 1995.

17. http://www.who.int/gb/EB_WHA/PDF/WHA55/ sa5517.pdf. Envejecimiento y Salud. Segunda Asamblea Mundial sobre Envejecimiento, Madrid, abril 2002. 


\section{Anexo 1}

Resumen: escala de evaluación de autonomía funcional

№ ficha evaluación:

Nombre:

Edad:

Domicilio:

Vive Con:

Solo $=$

Pareja $=$

Familia Nuclear=

Familia Extendida=

Otros $=$

Comunicación

\begin{tabular}{|l|l|l|l|l|}
\hline Puntaje & 0 & 1,0 & 2,0 & 3,0 \\
\hline Visión & & & & \\
\hline Audición & & & & \\
\hline Conversación & & & & \\
\hline Total & & & & \\
\hline
\end{tabular}

Funciones Mentales

\begin{tabular}{|l|l|l|l|l|}
\hline Puntaje & 0 & 1,0 & 2,0 & 3,0 \\
\hline 1. Memoria & & & & \\
\hline 2. Orientación & & & & \\
\hline 3. Comprensión & & & & \\
\hline 4. Juicio & & & & \\
\hline 5. Comportamiento & & & & \\
\hline Total & & & & \\
\hline
\end{tabular}

\section{Niveles de la escala de evaluación (SMAF) Modificada}

Los niveles se definen de la siguiente manera:

0-0,9: Persona totalmente independiente, no requiere que nadie lo ayude a desempeñar las labores, entiende perfectamente las instrucciones, se comunica a través de un lenguaje común, fácilmente entendible, presenta una buena orientación.

1,0-1,9: Dependencia leve: Con leves problemas de visión, audición, lenguaje, memoria, orientación pero que no le dificulta realizar sus actividades, en las cuales participa activamente.

2,0-2,9: Dependencia moderada: Persona que requiere de ayuda al realizar las actividades, pero puede caminar, sube y baja escaleras con ayuda o apoyo, tiene bastantes dificultades visuales, auditivas y de lenguaje pero pueden comunicarse, presentan serios lapsos de memoria, sólo están orientados en los eventos inmediatos, pueden presentar alteraciones del comportamiento (confusiones).

3.0: Dependencia severa: Persona postrada totalmente dependiente, no controla esfínteres, incapaz de comunicarse con su medio.

\section{COMUNICACIÓN}

1. Visión

\begin{tabular}{|l|lc|}
\hline 0 & Ve adecuadamente con o sin lentes corrientes \\
\hline 1 & Con problemas visuales pero ve lo suficiente para hacer las AVD \\
\hline 2 & Sólo ve contornos de los objetos y necesita supervisión en las AVD \\
\hline 3 & Ciego & lentes correctivos \\
\hline
\end{tabular}

AVD: actividades de la vida diaria. 


2. Audición
\begin{tabular}{|l|l|}
\hline 0 & $\begin{array}{l}\text { Escucha adecuadamente con o sin audífonos } \\
\text { Escucha si le hablan en voz alta }\end{array}$ \\
\hline 1 & Necesita que otra persona le coloque el audífono \\
\hline 2 & $\begin{array}{l}\text { Sólo escucha a gritos o ciertas palabras } \\
\text { Lee los labios }\end{array}$ \\
\hline 3 & Sordera e incapacidad para comprender qué es lo que le dicen a él o a ella \\
\hline
\end{tabular}

\section{Conversación}

\begin{tabular}{|l|l|}
\hline 0 & Se comunica verbalmente con el lenguaje común y fácilmente entendible \\
\hline 1 & Tiene problemas al conversar en el lenguaje pero es capaz de expresarse por sí mismo \\
\hline 2 & Tiene problemas de lenguaje para conversar pero es capaz de expresar sus necesidades \\
\hline 3 & Incapaz de comunicarse verbalmente \\
\hline
\end{tabular}

\section{FunCiONES MENTALES}

1. Memoria
\begin{tabular}{|l|l|}
\hline 0 & Memoria normal \\
\hline 1 & $\begin{array}{l}\text { Déficit menor de memoria reciente (nombres, compromisos) pero recuerda eventos } \\
\text { importantes }\end{array}$ \\
\hline 2 & $\begin{array}{l}\text { Serios lapsos de memoria (aislarse, tomar medicamentos, poner cosas fuera de su lugar, } \\
\text { comidas, visitas) }\end{array}$ \\
\hline 3 & Pérdida casi total de la memoria o amnesia \\
\hline
\end{tabular}

2. Orientación
\begin{tabular}{|l|l|}
\hline 0 & Orientado en el tiempo y espacio y persona \\
\hline 1 & Algunas veces desorientado en el tiempo, lugar y persona \\
\hline 2 & $\begin{array}{l}\text { Sólo orientado para eventos inmediatos (es decir, la hora del día) y en el ambiente de } \\
\text { vida habitual y con personas familiares }\end{array}$ \\
\hline 3 & Completamente desorientado \\
\hline
\end{tabular}

3. Comprensión

\begin{tabular}{|l|l|}
\hline 0 & Entiende instrucciones y solicitudes \\
\hline 1 & Lento para entender instrucciones y solicitudes \\
\hline 2 & Parcial entendimiento aun después de repetir instrucciones \\
\hline 3 & Ignorante de lo que ocurre alrededor de él o ella \\
\hline
\end{tabular}

\begin{tabular}{|l|l|} 
4. Juicio & \\
\hline 0 & Evalúa las situaciones y toma decisiones importantes \\
\hline 1 & Evalúa situaciones pero necesita ayuda al tomar decisiones importantes \\
\hline 2 & $\begin{array}{l}\text { Evalúa pobremente las situaciones y sólo toma decisiones importantes con una fuerte } \\
\text { sugerencia (indicación) }\end{array}$ \\
\hline 3 & Incapaz de evaluar o tomar decisiones y depende de otros para tomar decisiones \\
\hline
\end{tabular}

\section{Comportamiento}

\begin{tabular}{|l|l|}
\hline 0 & Apropiado comportamiento \\
\hline 1 & $\begin{array}{l}\text { Problemas de comportamiento menores (lloriqueos, labilidad emocional, terquedad). } \\
\text { Supervisión ocasional }\end{array}$ \\
\hline 2 & $\begin{array}{l}\text { Problema mayor de comportamiento que requerirá constante supervisión (agresividad } \\
\text { hacia él u otros, disturbios o confusiones) }\end{array}$ \\
\hline 3 & Peligroso, requiere restricción (encierro). Daña a otros o autodestrucción. Trata de fugarse \\
\hline
\end{tabular}

